Maaswinkel-Mooij $\mathrm{P}$, et al. Mental retardation and behavioral problems as presenting signs of a creatine synthesis defect. Ann Neurol April 2000;47:540543). (Respond: Dr van der Knaap, Department of Child Neurology, Free University Hospital, PO Box 7057, 1007 MB Amsterdam, The Netherlands).

COMMENT. A deficiency of guanidinoacetate methyltransferase, an inborn error of metabolism of creatine synthesis, results in a decrease in body creatine, accumulation of guanidinoacetate, and decreased urinary excretion of creatine. This rare syndrome of creatine synthesis defect is characterized by psychomotor developmental delay or regression, behavioral abnormalities, muscle hypotonia, extrapyramidal movements, and intractable epilepsy. Diagnosis is suspected when urine amino acids and organic acids are increased generally, relative to creatine. Treatment with creatine monohydrate is followed by improvement but not complete resolution of symptoms.

\title{
BECTS WITH INTRACTABLE SEIZURES AND COGNITIVE DECLINE
}

Two children, aged 2 and 4 years, with a characteristic onset of benign childhood epilepsy with centrotemporal spikes (BECTS), developed intractable seizures and cognitive decline, as reported from the Cleveland Clinic Foundation, $\mathrm{OH}$. Seizures were repetitive clonic in one and hemifacial clonic in the second patient, and occurred more than 100 times daily. Some nocturnal generalized tonic-clonic seizures also occurred. Ictal EEGs showed focal sharp waves in central midline or centrotemporal regions, with or without evolution to generalized discharges. Interictal epileptiform, focal sharp wave, discharges were activated during drowsiness and sleep. MRIs were normal. Despite trials with five different antiepileptic drugs, including valproic acid $(120 \mathrm{mcg} / \mathrm{ml})$, carbamazepine, phenobarbital, phenytoin, ethosuximide, with prednisone and acetazolamide, seizures were uncontrolled. The IQ declined from 139 at 6 years, to 103 at 8 years, when surgery was considered but deferred in one. Seizures resolved spontaneously after 9 years of age in one, and they improved with the ketogenic diet after age 5 years in another. Cognition improved after seizures were controlled, or resolved spontaneously, in both patients. (Ong H-T, Wyllie E. Benign childhood epilepsy with centrotemporal spikes: Is it always benign? Neurology March (1 of 2) 2000;54:1182-1185). (Reprints: Dr Elaine Wyllie, Pediatric Epilepsy Program S51, The Cleveland Clinic Foundation, 9500 Euclid Ave, Cleveland, OH 44195).

COMMENT. BECTS is usually a benign disorder, characterized by infrequent seizures, with or without therapy, and spontaneous remission in later childhood. Rarely, seizures may be repetitive and frequent, refractory to medication, and associated with intellectual regression. The authors stress the need for a conservative approach to management, since spontaneous remission can be expected. The role of the antiepileptic medication in the cognitive decline of one of these patients is certainly suspect.

\section{HIGH-DOSE IV STEROIDS IN LANDAU-KLEFFNER SYNDROME}

Intravenous therapy with methylpredisolone $(20 \mathrm{mg} / \mathrm{kg}$ daily $)$ was effective in two six-year-old children with Landau-Kleffner syndrome treated at Jichi Medical School, Tochigi, Japan. A combination of valproate and a benzodiazepine had previously resulted in decreased seizures and EEG improvement, but speech and language were not improved. After 3 consecutive daily treatments with high-dose IV corticosteroids, speech was increased and receptive language improved. After a third course of therapy, the children could speak in sentences, and their subsequent language development was sustained. 